Revista Digital Universitaria

Vol. 23, Núm. 1, enero-febrero 2022

\title{
Modalidad abierta, a distancia y mixta: mecanismo para disminuir la desigualdad educativa
}

\author{
Laura C. Casillas Valdivia
}

\begin{abstract}
Resumen
En México, la cobertura en educación superior es altamente heterogénea y desigual. Al ser la Ciudad de México la que concentra el mayor número de Instituciones -públicas y privadas- y planes y programas de estudio, se deja con limitado acceso a personas que radican en otras entidades federativas; o bien, a personas que por diversas razones no pueden asistir de manera presencial. En este sentido el SUAYED de la unAM, con la experiencia acumulada de 50 años de ofrecer educación superior de calidad, funge como mecanismo para disminuir esta desigualdad, al absorber el 11\% de la matrícula que cursa estudios de licenciatura en modalidades no presenciales en instituciones públicas de educación superior.
\end{abstract}

Palabras clave: educación superior, instituciones, planes de estudio, TIc, educación a distancia

\section{OPEN, DISTANCE AND MIXED MODALITY: MECHANISM TO REDUCE EDUCATIONAL INEQUALITY}

\begin{abstract}
In Mexico, higher education coverage is highly heterogeneous and unequal. As Mexico City concentrates the largest number of Institutions - public and private- and study plans and programs, it leaves limited access to people who live in other states, or to people who for various reasons cannot attend in person. In this sense, UNAM 'S SUAYED, with the accumulated experience of 50 years of offering quality higher education, serves as a mechanism to reduce this inequality by absorbing $11 \%$ of the enrollment that attends undergraduate studies in non-face-to-face modalities in public universities.
\end{abstract}

Keywords: higher education, institutions, curricula, ict, distance education 
Jefa de la División Sistema Universidad Abierta y Educación a Distancia, Facultad de Economía, unam. básico, sólo 39 llegan a programas de educación superior y apenas 26 logran concluir sus estudios.

Entre 2018 y 2020 el porcentaje de población con rezago educativo a nivel nacional mantuvo valores cercanos al 19\%; sin embargo, se hizo evidente un aumento del porcentaje de la población de 3 a 21 años que no asiste a la escuela y no cuenta con la educación obligatoria. Llama la atención el hecho de que el porcentaje de la población de 3 a 21 años que no asiste a la escuela y no cuenta con educación obligatoria (educación media superior) aumentó en todos los deciles de ingreso, lo que lleva a pensar que el mayor problema es la cobertura (CONEVAL, 2021, p.11). En el país, en educación superior, la cobertura alcanza apenas el 34.4\% de jóvenes entre 18 y 22 años, cuestión que complica el desarrollo y bienestar de la población.

Si bien esta información apenas ofrece un escenario parcial, es notorio que un segmento importante de la población se encuentra excluido del sistema educativo nacional. La matrícula en educación superior sigue estando concentrada en las principales metrópolis del país y la disponibilidad de espacios crece de manera muy lenta (Fuentes, 2017). Las universidades —en particular las públicas - no cuentan con la capacidad para atender la creciente demanda de ingreso de un número significativo de aspirantes. Por otra parte, la Ciudad de 
México cuenta con una situación de privilegio en relación con el resto del país en cuanto a oferta educativa, cobertura y años de escolaridad, lo que se expresa en desigualdades, heterogeneidad en la calidad y altos costos de enseñanza en las instituciones privadas de educación superior (Solís, 2017).

Aunado a lo anterior, la emergencia sanitaria derivada de la covID-19 obligó a un cambio en la dinámica de trabajo del sistema educativo a nivel mundial, haciendo más evidentes las múltiples e inaceptables formas de desigualdad y violencia que padecen nuestras sociedades (Graue, 2020).

Ante esta situación, según la Encuesta para la Medición del Impacto coviD-19 en la Educación (ECOVID-ED) 2020, de 54.3 millones de personas de entre 3 y 29 años, el 62\% - poco mas de 33.5 millones - se encontraban inscritas en alguna actividad académica durante el ciclo escolar 2019 - 2020. Sin embargo, por motivos asociados al covid-19 (2.3 millones de personas) o por falta de dinero o recursos (2.9 millones de personas), un total de $\mathbf{5 . 2}$ millones de personas (de las cuales 2.5 son mujeres) no se inscribieron al ciclo inmediato 2020-2021.

Es, ante este escenario, que las modalidades educativas no presenciales (abierta, a distancia o mixta), adquieren relevancia como una alternativa real para atender la emergencia sanitaria. En los más recientes años el porcentaje de alumnos que cursan sus estudios en modalidad no presencial se ha incrementado de manera constante. Mientras que en el año 2014 representaba el 13\% de la matrícula nacional, para el ciclo escolar 2020 se elevó al 19\% del total.

Figura 1. Matrícula inscrita a nivel superior por modalidad. Nota: Elaborado con datos de Principales Cifras del Sistema Educativo Nacional, varios años. Dirección General de Planeación, Programación y Estadística Educativa de la SEP (https:// www.planeacion.sep.gob.mx/ estadisticaeindicadores.aspx).

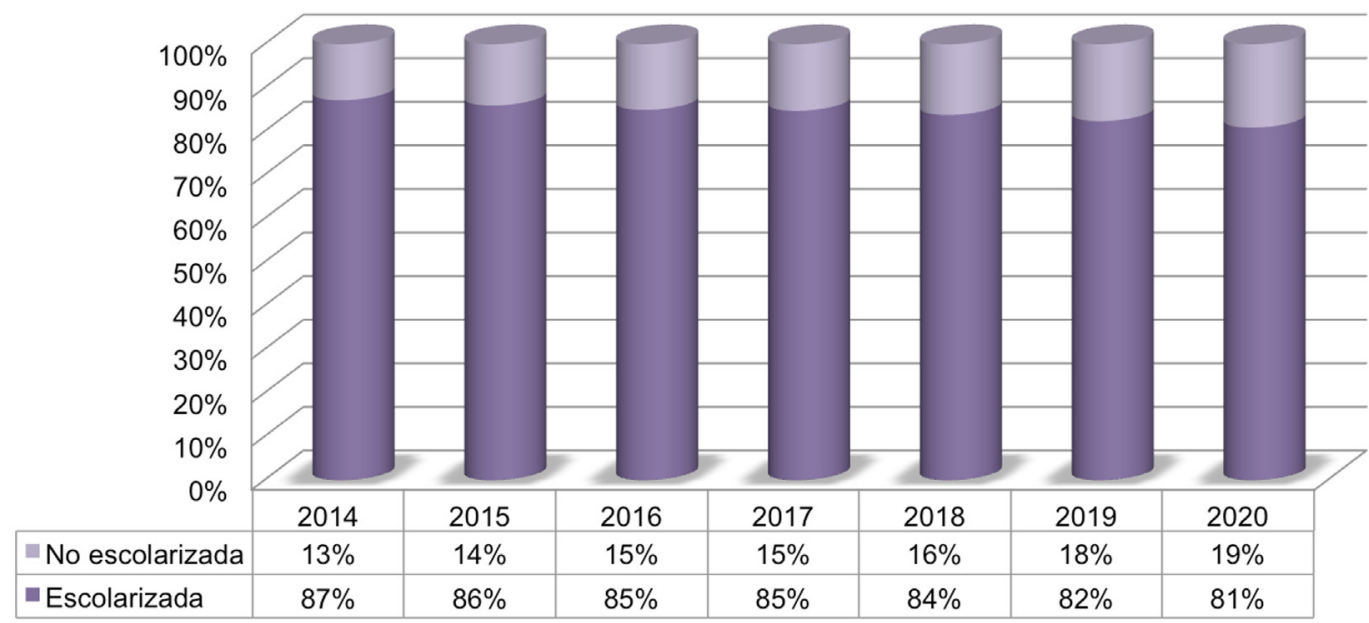

Destaca que la figura 1 muestra que, durante el periodo de pandemia, la modalidad escolarizada tuvo un ligero decremento de su matrícula (-0.76\%), mientras que en la no presencial continuó con su trayectoria ascendente (+9.55\%), lo que refuerza el potencial de esta modalidad ante situaciones adversas. No obstante, aún queda un largo camino por recorrer, ya que para el ciclo escolar 2020-2021, del alumnado total inscrito a nivel nacional en educación superior en modalidad no presencial, sólo el 37\% lo está en instituciones públicas. 
Figura 2. Alumnado inscrito en educación superior, en instituciones públicas y privadas, por modalidad. Nota: Elaborado con datos de Principales Cifras del Sistema Educativo Nacional 2020-2021. Dirección General de Planeación, Programación y Estadística Educativa de la SEP (https://www.planeacion.sep. gob.mx/estadisticaeindicadores. aspx).

Figura 3. Porcentaje de alumnado atendido, en instituciones públicas, en modalidad no presencial. Nota: Elaborado con datos de Principales Cifras del Sistema Educativo Nacional 2020-2021. Dirección General de Planeación,

Programación y Estadística

Educativa de la SEP (https:// www. planeacion.sep.gob.mx/ estadisticaeindicadores.aspx) y Agenda Estadística Universitaria 2021, UNAM (https://www. estadistica.unam.mx/agenda. php).

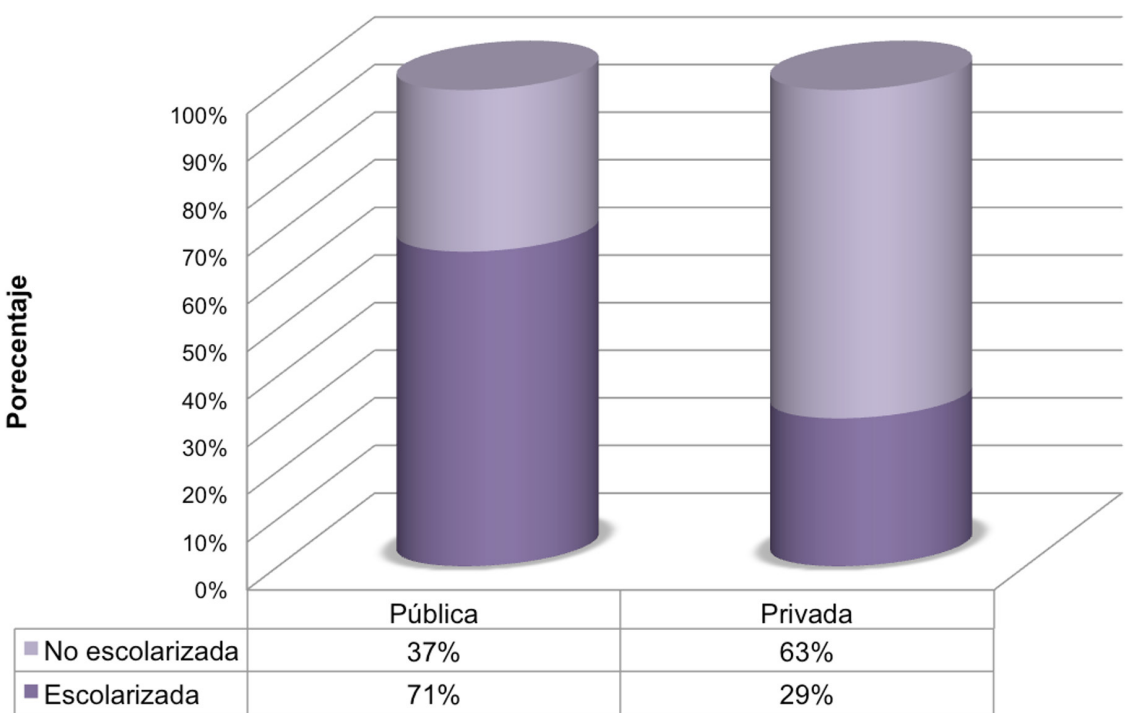

Lo anterior deja de manifiesto que, a nivel nacional, las opciones educativas para la población que por diversas razones no puede acudir de forma presencial a las universidades públicas resultan limitadas. Y dadas las condiciones económicas del país, apenas unos cuantos tienen la opción de pagar educación superior privada.

En este sentido, la Universidad Nacional Autónoma de México (UNAm), en su carácter de institución pública y referente a nivel nacional por su posición en los rankings nacionales e internacionales atiende, en sus 50 planes de estudio, atiende actualmente a aproximadamente el $11 \%$ de la matrícula nacional que cursa sus estudios de licenciatura en modalidad no presencial en instituciones públicas de educación superior.

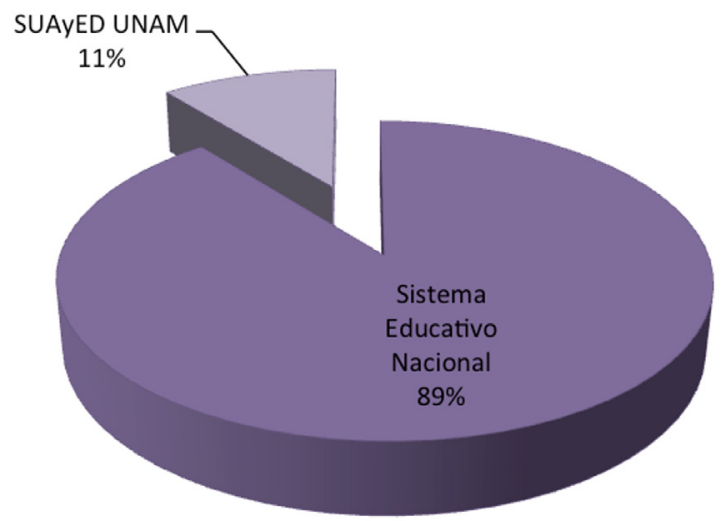

Por su parte, de acuerdo con la Agenda Estadística 2020-2021 de la UNAM (UNAM, DGPL, 2020), en el ciclo escolar 2020-2021, el alumnado inscrito asciende a 217,808 personas, de las cuales 37,314 (17\%) realizan sus estudios en la modalidad no presencial. Históricamente, la Máxima Casa de Estudios ha sido pionera en los programas educativos de nivel superior en sus modalidades 
Figura 4. Matrícula unAm por modalidad

Nota: En la gráfica se muestra

la matrícula de la UnAm por modalidad en diferentes periodos, los datos completos se pueden consultar en el Portal de Estadística Universitaria de la Universidad Nacional Autónoma de México (https://www. estadistica.unam.mx/agenda. abierta y a distancia. Primero, a través de la creación del Sistema de Universidad Abierta (suA) hace 50 años, y después con la implementación de la modalidad a distancia (SED), que juntas consolidan el Sistema de Universidad Abierta y Educación a Distancia (SUAYED).

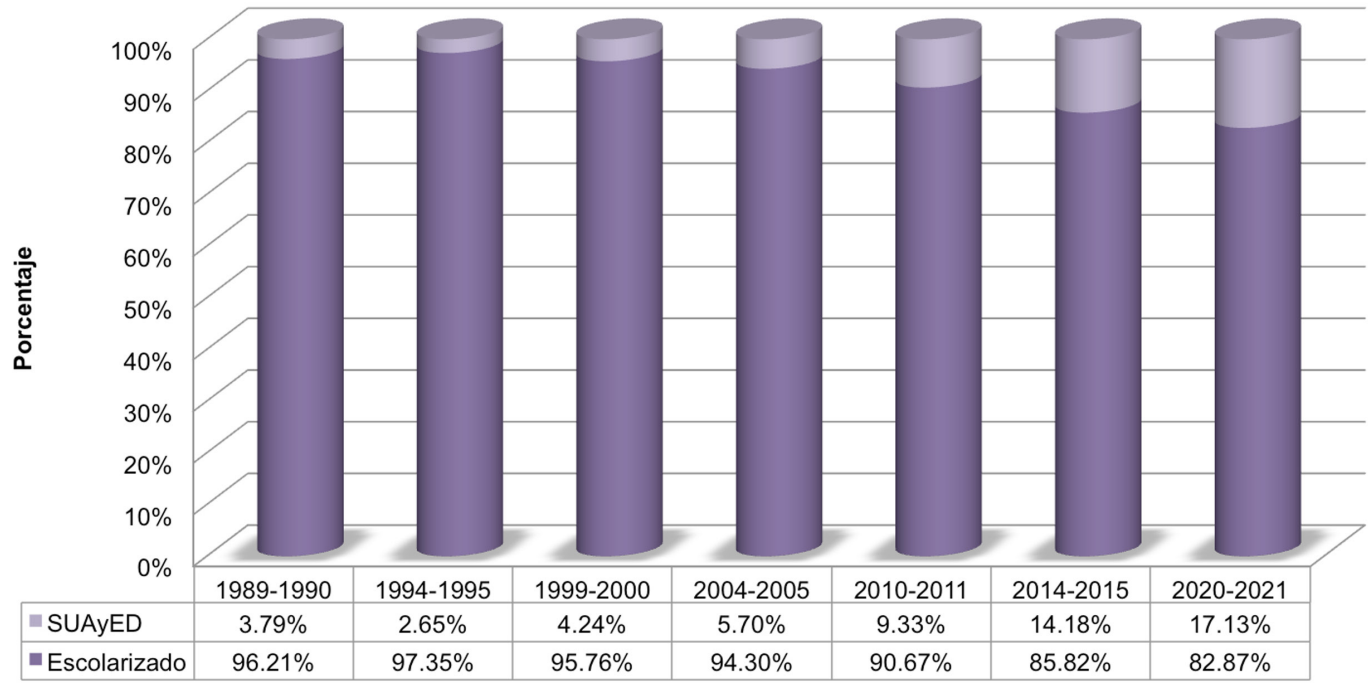

En los últimos 20 años, el sUAYED unAm, pasó de atender al 3.79\% de la matrícula total de la UNAM, a poco más del 17\%. De esta forma, ha demostrado que tiene la capacidad de brindar una opción educativa inclusiva y de calidad que permite atender a segmentos de población que no están en posibilidad de asistir de forma presencial, ya sea por cuestiones laborales o familiares, o bien porque en su lugar de residencia no existen instituciones públicas que permitan cursar estudios a nivel licenciatura en una modalidad no presencial. De este modo se reducen las desigualdades en acceso a la educación que padecen las diferentes entidades educativas de la República Mexicana.

En este contexto, la unAm a través del SUAYED, tiene ante sí los retos de: a) aumentar la cobertura de planes de estudio impartidos en modalidad no presencial, b) definir un modelo educativo para las modalidades mixtas y c) en general ofrecer un mayor número de alternativas de educación que integren herramientas tecnológicas y de comunicación tanto de planes de estudio formalmente constituidos, como de actividades académicas abiertas a toda la población, en el entendido de que educar con calidad es un proceso único, independiente de la modalidad que se siga para llevarlo a cabo. Y este proceso implica formar seres humanos libres, capaces y útiles a la sociedad, bajo el precepto de que la educación es un bien público y no una mercancía.

\section{Referencias}

* coneval (2021). Nota técnica sobre el rezago educativo, 2018-2020. https://cutt.ly/ IljnDGm 
"Modalidad abierta, a distancia y mixta: mecanismo para disminuir la desigualdad educativa" Laura C. Casillas Valdivia Vol. 23, Núm. 1, enero-febrero 2022 Revista Digital Universitaria

* DGPl. (1989-1990). Agenda y anuarios estadísticos unam.

* DGPL. (1994-1995). Agenda y anuarios estadísticos unam.

* DGPl. (1999-2000). Agenda y anuarios estadísticos unam.

* DGPL. (2004-2005). Agenda y anuarios estadísticos unam.

* DGPL. (2010-2011). Agenda y anuarios estadísticos unam.

* DGPl. (2014-2015). Agenda y anuarios estadísticos unam.

* DGPl. (2020-2021). Agenda y anuarios estadísticos unam.

* Graue, E. (2019). Plan de Desarrollo Institucional, unam 2019-2023. unam.

* Dirección General de Planeación, Programación y estadística Educativa (2021) Principales cifras del sistema educativo nacional 2020-2021. SEP.

* Fuentes, L. (2017). Educación superior. La desigualdad. https://cutt.ly/yljnZ08

* InEGI. (2020). Censo de Población y Vivienda. https://cutt.ly/TljnCce

* ineGi. (2021). Encuesta para la Medición del Impacto covid-19 en la Educación (ECOVID-ED) 2020.

* Solís, P. (2014). Desigualdad social y efectos institucionales en las transiciones educativas. En E. Blanco, P. Solís \& H. Robles (coord.), Caminos desiguales. Trayectorias educativas y laborales de los jóvenes en la Ciudad de México (71-106). El Colegio de México-IneE.

\section{Cómo CITAR ESTE ARTículo}

* Casillas, Laura. (2022, enero-febrero). Modalidad abierta, a distancia y mixta: mecanismo para disminuir la desigualdad educativa. Revista Digital Universitaria (RDU), 23, (1). http://doi.org/10.22201/cuaieed.16076079e.2022.23.1.10 